J. Orn. 126, 1985: S. 273-279

Aus dem Lehrstuhl für Verhaltensphysiologie, Universität Bielefeld

\title{
Der Anteil akustischer Komponenten an der Auslösung der Balz männlicher Zebrafinken (Taeniopygia guttata castanotis)
}

\author{
Von Hans-Joachim Bischof
}

\section{Einleitung}

Nach Isolation balzen männliche Zebrafinken in der Regel bei Anwesenheit eines ․ Eine Reihe von Untersuchungen hat gezeigt, daß an der Auslösung der Balz vorwiegend optische Parameter beteiligt sind, akustische Äußerungen des $\&$ dagegen eine untergeordnete Rolle spielen. So werden die $\sigma^{t}$ auch durch ausgestopfte Weibchenattrappen zur Balz stimuliert (IMmELMANN 1959). Selbst zweidimensionale Abbildungen von $\$$ lösen Balzverhalten aus (Bischof 1980). Die Häufigkeit und Ausprägung der Balzsequenzen hängt dabei offenbar davon ab, wie ähnlich die gebotene Attrappe einem normal gefärbten, lebenden Weibchen ist (BIschof 1980, GARson et al. 1980, Bischof et al. 1981).

Lebende $P$ sind in jedem Fall wesentlich wirksamere Auslöser als zwei- oder dreidimensionale Attrappen. Eine Veränderung einzelner optischer Merkmale, wie zum Beispiel die Schwarzfärbung des Schnabels, die bei Attrappen zu einer signifikanten Reduktion der Balzhäufigkeit führt, wirkt sich bei lebenden Vögeln nur geringfügig aus (Garson et al. 1980, Bischof 1983). Immelmann (1959) vermutet, daß das ơ gegenüber Attrappen mit der Balzkette nicht fortfahren kann, da die entsprechenden Reaktionen des Weibchens fehlen. Morris (1954) führt aus, daß besonders die sogenannte „Verbeugung“ des $\%$ eine wichtige auslösende Komponente darstellt. Diese Vermutung konnte von Garson et al. (1980) experimentell belegt werden.

Ungeklärt ist bisher, wie weit Lautäußerungen der $\$$ die Auslösung der Balz des $\sigma^{*}$ beeinflussen. Stimmfühlungslaute werden von $\$$ ständig, auch während der Balz, geäußert. Es ist kaum anzunehmen, daß diese Laute nicht dazu beitragen, die Wahrscheinlichkeit für die Balz der ơ zu erhöhen. Wie stark dieser Einfluß ist, sollte in dieser Untersuchung geklärt werden. Dazu wurde in zwei verschiedenen Experimenten die Häufigkeit verschiedener Balzelemente bei rein akustischem und bei rein optischem Kontakt mit derjenigen bei kombinierter Darbietung beider Reizmodalitäten verglichen.

\section{Versuchsvögel und Methoden}

Experiment 1 wurde mit 8 an Einzelkäfighaltung gewöhnten domestizierten Zebrafinken- $\sigma^{x}$ aus den Zuchten des Lehrstuhls durchgeführt. Für alle Tests wurde dasselbe $q$ verwendet. Die Versuchsanordnung bestand aus zwei nebeneinanderstehenden Käfigen, die durch eine Wand optisch getrennt werden konnten. Die $\sigma^{\prime}$ wurden für jeweils $15 \mathrm{~min}$ in zufälliger Reihenfolge in drei verschiedenen Testsituationen beobachtet: 
1. ohne 9 im zweiten Käfig (A);

2. mit 9 im zweiten Käfig, optisch isoliert, akustischer Kontakt war möglich (B);

3. mit $९$ im zweiten Käfig, optischer und akustischer Kontakt möglich. (C).

Zwischen den Versuchen lagen jeweils drei Tage, in denen das ơ von $\$$ optisch und akustisch isoliert gehalten wurde. Folgende Verhaltensweisen (nach MorRIs 1954) wurden mit Hilfe eines Eventrecorders (Fa. Peiseler) registriert:

a) Schnabelwetzen;

b) ein prägnanter Kontaktlaut (Laut III), der in der Frequenzzusammensetzung dem lauten Distanzruf der Vögel ähnelt, aber kürzer und leiser ist;

c) Verbeugen vor dem $\%$;

d) Wendesprung $\left(180^{\circ}\right.$-Drehung auf der Stange bei Annäherung an das Weibchen) mit $90^{\circ}$-Drehung des Körpers;

e) Anzahl der Motive des Gesangs.

Experiment 2 mit 8 anderen Zebrafinken- $0^{*}$ : Der zweite Käfig mit dem $q$ befand sich bei diesem Versuch in einer schallisolierten Kammer ( Fa. Getra). Das ot hatte optischen Kontakt zum $\&$ durch ein in der Frontseite der Kammer befindliches Fenster. Im ersten Test wurde die Kammer geschlossen, so daß nur optischer Kontakt möglich war (Vers.-sit. D), im zweiten Test wurde die Tür der Kammer geöffnet, so daß die Tiere sich gegenseitig hören und sehen konnten (Vers.-sit. E, vergleichbar mit C von Vers. 1). Registriert wurden, wieder für jeweils $15 \mathrm{~min}$, die gleichen Verhaltensweisen wie in Experiment 1.

$\mathrm{Da}$ die Vögel individuell sehr unterschiedlich auf die verschiedenen Situationen reagierten, wurde auf die Berechnung von Mittelwerten verzichtet; die Abbildungen zeigen die direkt den Versuchsprotokollen entnommenen Einzelwerte. Unterschiede zwischen den einzelnen Testsituationen wurden mit dem Vorzeichentest (zweiseitig, SIEGEL 1976) auf ihre Signifikanz überprüft.

Ich danke Herrn J. BöHner für die kritische Durchsicht des Manuskripts und eine Reihe von. fruchtbaren Anregungen und Hinweisen. Frau E. GeIssLer danke ich für die Anfertigung der Zeichnungen. Einige Mitglieder des Blocks „Verhaltensphysiologie“ halfen bei der Erhebung der Daten. Auch ihnen sei herzlich gedankt. Mit Unterstützung der Deutschen Forschungsgemeinschaft (Bi 245/3).

\section{Ergebnisse}

Experiment 1 (Abb. 1):

a) Unterschiede zwischen Situation A (ohne \$) und B (nur akustischer Kontakt mit $\operatorname{dem}$ \%): "Schnabelwetzen“ und „Laut III" traten in beiden Situationen auf. Laut III wurde in Situation B signifikant häufiger gezeigt $(p<0,03)$. Schnabelwetzen war nicht signifikant unterschiedlich, Gesang trat in Situation A einmal auf, in Situation B dreimal (n. s.). Wendesprünge und Verbeugungen waren in beiden Situationen nicht zu beobachten.

b) Unterschiede zwischen Situation B (nur akustischer Kontakt mit dem $q$ ) und C (akustischer und optischer Kontakt): "Schnabelwetzen" trat in Versuch C nicht signifikant häufiger auf als in B (n. s.). Laut III trat in C seltener auf als in B (n. s.). Verbeugungen, Wendesprünge und die Anzahl der geäußerten Motive erhöhten sich in $C$ signifikant $(p<0,008)$ gegenüber $B$. Dabei traten, wie oben erwähnt, Wendesprünge und Verbeugungen in B nicht auf; Gesang äußerten nur 3 Vögel.

Experiment 2 (Abb. 2):

Unterschiede zwischen Versuch D (nur optischer Kontakt) und E (akustischer und optischer Kontakt): „Schnabelwetzen“ änderte sich von Situation D zu E nicht signifi- 
kant, Laut III trat signifikant häufiger auf $(\mathrm{p}<0,07)$, Verbeugen und Wendesprünge änderten sich nicht signifikant. Die Anzahl der Motive vergrößerte sich von D zu E signifikant $(\mathrm{p}<0.032)$.

\section{Diskussion}

Die Häufigkeit und Intensitäten der einzelnen Verhaltensweisen sind sicher durch die Versuchsbedingungen beeinflußt. Sowohl die räumliche Trennung des ơ vom ? und die durch Käfigstäbe behinderte Sicht, als auch die Enge der Käfige und die Anwesenheit eines Beobachters sollten sich hemmend auf die Verhaltensweisen auswirken. Da sämtliche Tests jedoch unter gleichen Bedingungen ausgeführt wurden und jeweils die individuellen Werte der einzelnen Vögel miteinander verglichen wurden, ist eine Auffassung über die Beteiligung optischer und akustischer Parameter an der Auslösung der Balz des Zebrafinkenmännchens sicher erlaubt.

„Schnabelwetzen“ und „Laut III“ treten bei Zebrafinken auch unabhängig von der Balz auf. Die ursprüngliche Bedeutung des Schnabelwetzens ist im Komfortverhalten zu suchen; es ist jedoch auch eine besonders bei sexueller Erregung häufig zu beobachtende Übersprungbewegung (vgl. Morris 1954). Möglicherweise ist das Schnabelwischen auch schon bis zu einem gewissen Grad als fester Bestandteil in die Balz hineinritualisiert. Laut III kann als Indikator für eine allgemeine Erregung gewertet werden. Er tritt z. B. auf beim Betreten einer Voliere, bei Annäherung an den Käfig eines einzeln gehaltenen Vogels oder bei Annäherung an Nester während der Brutzeit (BöHNER, pers. Mitt.).

„Verbeugen“, „Wendesprünge“ und „Gesang" sind Elemente des Balzverhaltens (Immelmann 1959, 1962, Morkis 1954). Neben dem in der Balz geäußerten gerichteten Gesang tritt allerdings auch eine geringfügig veränderte Gesangsform (ungerichteter Gesang) außerhalb der Balz auf (Immelmann 1959, 1962, Sossinka \& Böhner 1980). Eine Unterscheidung zwischen beiden Typen wurde hier nicht vorgenommen. Dic Verbeugung wird der sogenannten Balzeinleitung zugerechnet (MORRIS 1954, IMMELMANN 1959, 1962), während die Wendesprünge zum auf die Balzeinleitung folgenden Balztanz gezählt werden. Der Balzgesang wird vorwiegend während des Balztanzes geäußert.

Die meisten Balzelemente werden bei Anwesenheit beider Komponenten, der optischen und der akustischen, ausgelöst. Bei Darbieten nur der optischen Komponente (D) zeigt sich bei „Verbeugen“ und "Wendesprung" kein signifikanter Unterschied zum Kontrollversuch mit beiden Reizen, während die Menge der geäußerten Motive gegenüber dem Versuch mit Darbietung optischer und akustischer Reize (E) signifikant niedriger ist. Vermutlich erhöht also der akustische Kontakt zum $q$ die Wahrscheinlichkeit der Auslösung des Balzgesangs, nicht aber die der übrigen Komponenten.

Im Gegensatz zum optischen Kontakt kann rein akustischer Kontakt offenbar die Balz des ot nicht auslösen. Bis auf den Gesang, der auch unter Versuchssituation (B) 

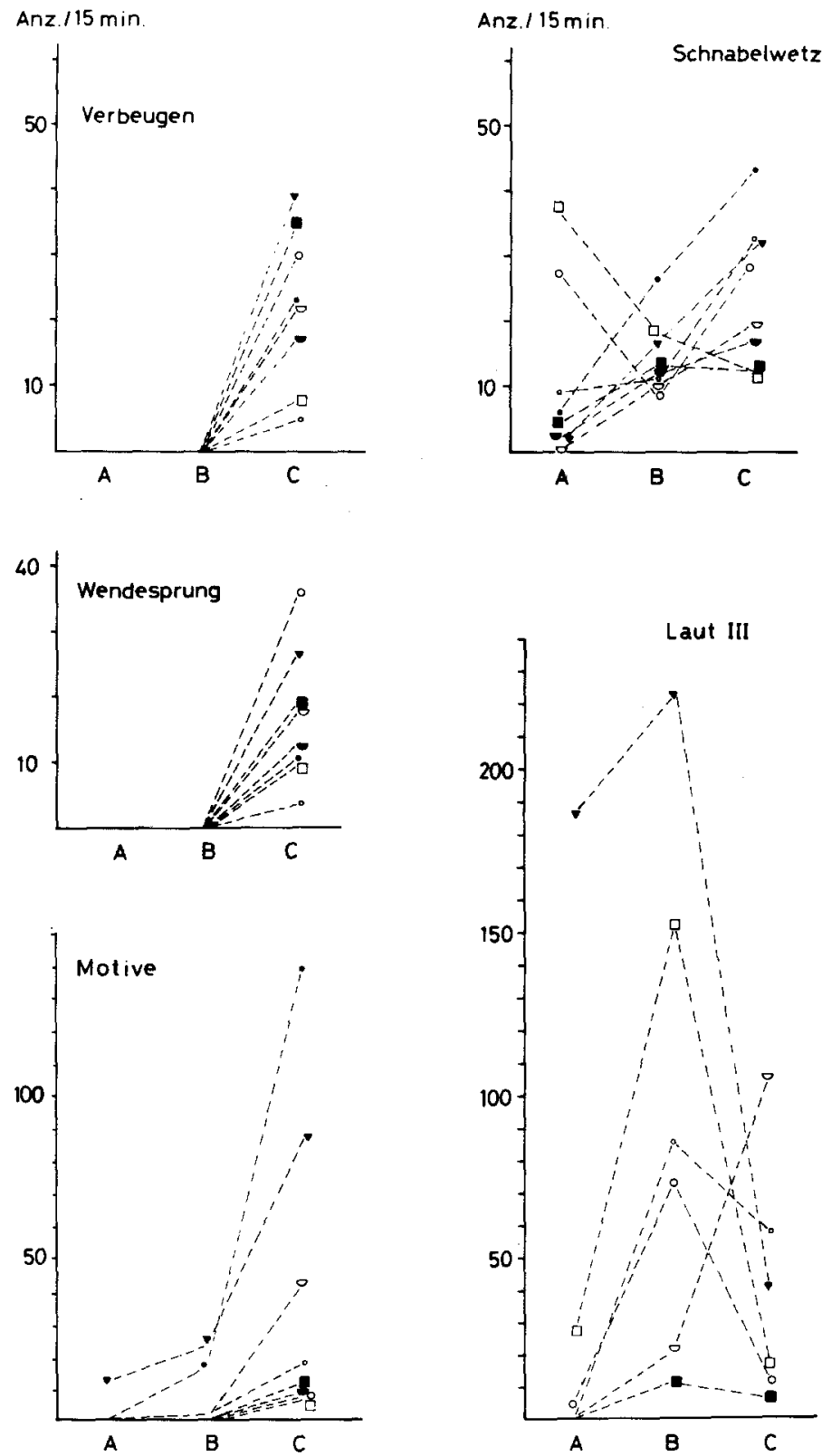

Abb. 1. Anzahl verschiedener, in drei unterschiedlichen Versuchssituationen gemessenen Verhaltensweisen von Zebrafinken- 9 . Abszisse: Anzahl Vhw pro 15 min. Vers.-sit. A: isolierter Vogel; B: akustischer Kontakt zum \% ; C: akustischer und optischer Kontakt. Für jeden Vogel wurde ein unterschiedliches Symbol verwendet. - Frequency of different behaviours in different experimental situations. Abszissa: no. of behavioural elements/15 min. A: isolated bird; B: acoustic contact to the female; $C$ : visual and acoustic contact to the female. "Verbeugen" $=$ bowing; "Wendesprung" = "zigzag" courtship dance; "Motive" $=$ motifs; "Schnabelwetzen" = bill wiping; "Laut III" = contact call III. 
Anz./15 min.
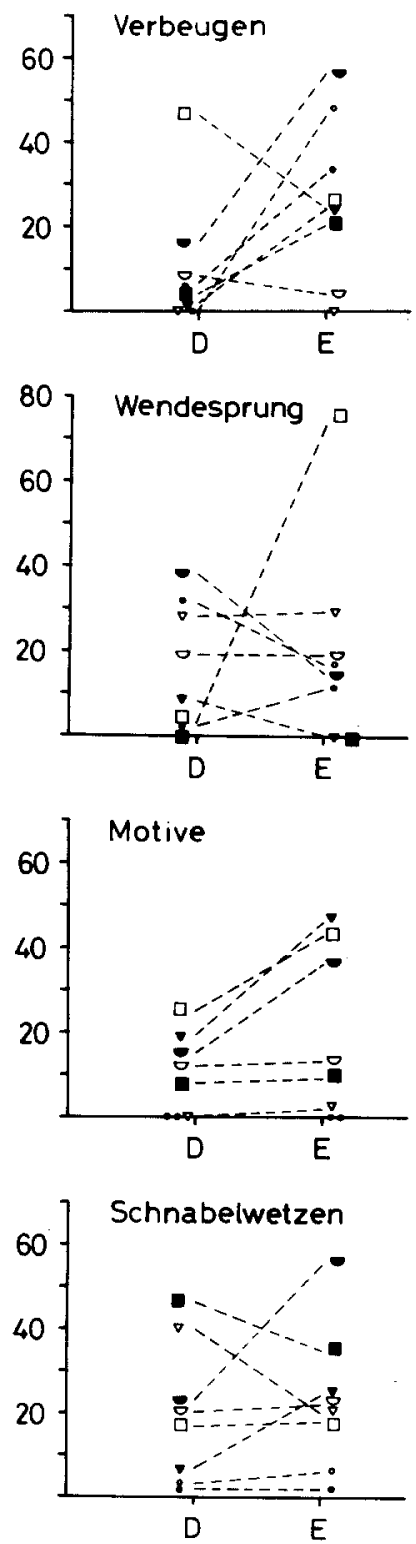

Anz./15 min.

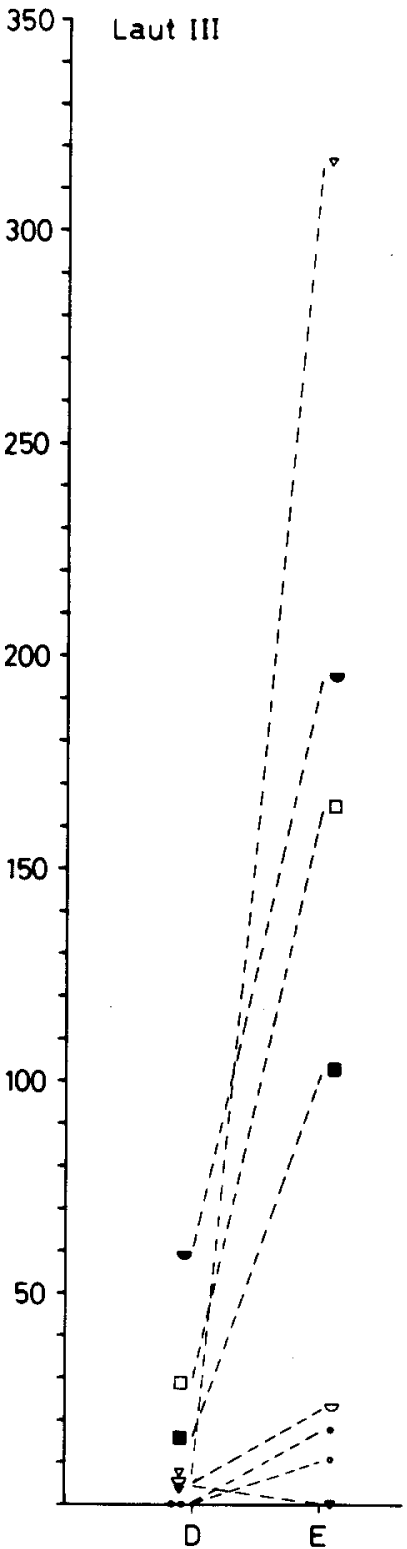

Abb. 2. Siehe Abb. 1. D: nur optischer Kontakt mit dem $\$$; E: akustischer und optischer Kontakt. - See fig. 1. D: visual contact to the female; E: visual and acoustic contact. 
auftrat, waren wie bei Anwesenheit des $\$$ (A) keine Balzverhaltensweisen zu beobachten, während in Situation (C) bei allen $\sigma^{t} \sigma^{t}$ alle registrierten Verhaltensweisen auftauchen. Da gleichzeitig keine anderen Balzverhaltensweisen auftraten, dürfte es sich bei den in Situation B vorgetragenen Motiven um ungerichteten Gesang handeln (vgl. SosSINKA \& BöHNer 1980). Der akustische Kontakt mit dem $\varsubsetneqq$ erhöht die Wahrscheinlichkeit, daß dieser Gesangstyp auftritt. Dies unterstützt die Hypothese, daß es sich beim ungerichteten Gesang um ein Sexualverhalten mit sehr niedriger Schwelle (z. B. Morris 1954, s. aber Caryl 1981) handelt, der im Gegensatz zu anderen Balzverhaltensweisen schon durch akustischen Kontakt mit einem $q$ stimuliert wird.

Obwohl die Ergebnisse für das „Schnabelwetzen“ keine signifikanten Unterschiede zeigen, sind Tendenzen klar zu erkennen. "Schnabelwetzen“ erhöht sich bei akustischem Kontakt gegenüber der Situation ohne $q$, erhöht sich weiter bei zusätzlichem Sichtkontakt mit dem $\$$ und ist geringer bei nur optischem Kontakt. Möglicherweise spiegelt diese Verhaltensweise den Erregungsgrad des Vogels wider, der ansteigt, je vollständiger der Kontakt mit dem $\$$ ist. Interessant im Zusammenhang mit der Übersprunghypothese wäre der Test, ob diese Verhaltensweise bei Aufhebung der räumlichen Trennung vom 9 zugunsten von Balzverhaltensweisen wieder abnimmt.

„Laut III" wird offenbar durch den akustischen Kontakt mit dem $\$$ erhöht. Bei rein optischem Kontakt wird dieser Laut zwar geäußert, wie auch in den Versuchen ohne Anwesenheit eines $F$, die Häufigkeit ist aber nicht sehr groß. Ordnet man den Laut III als Indikator für den Erregungszustand des Vogels ein (s. o.), so würde das bedeuten, daß der alleinige akustische Kontakt mit dem $\$$ - eine die „echten “ Balzverhaltensweisen nicht auslösende Situation - den allgemeinen Erregungsgrad des ot erhöht.

Akustischer Kontakt mit dem $P$ ist also allein nicht in der Lage, Balzverhaltensweisen des $\sigma$ auszulösen. Er bewirkt aber eine Erhöhung nicht balzspezifischer Verhaltensweisen wie „Schnabelwetzen“ oder „Laut III“, die wohl als Indikatoren für den allgemeinen Erregungszustand des Vogels anzusehen sind. Wegen dieses erhöhten Erregungszustandes erfolgen dann die Balzverhaltensweisen wie „Balzgesang“ bei kombiniertem optischem und akustischem Kontakt häufiger und intensiver als bei rein optischem Kontakt. Dasselbe wäre theoretisch für "Verbeugung" und „Wendesprung“" zu fordern. Bei diesen Verhaltensweisen ist zwar eine ähnliche Tendenz zu beobachten, die Ergebnisse sind aber nicht signifikant. Dies könnte eine Folge der Käfigbedingungen sein. Besonders der Wendesprung ist eine „bewegungsintensive" Verhaltensweise, die unter natürlichen Bedingungen als stereotyp wiederholte Bewegung im Rahmen des relativ viel Platz erfordernden Balztanzes zu beobachten ist (IMmelmann 1962, vgl. Morris 1954). Diese raumgreifenden Anteile der Balz (Springen von Ast zu Ast, Balz$\operatorname{tanz}$ ) sind unter Käfigbedingungen selten oder gar nicht zu sehen bzw. auf wenige Anteile, wie ein oder zwei einzelne Wendesprünge, reduziert. „Gesang "wird naturgemäß von diesen vor allem die Lokomotorik betreffenden Einschränkungen nicht berührt. In der Terminologie von BuchHolTz (1982) wären die Lautäußerungen des $\$$ unspezifische Reize, die zwar die Handlungsbereitschaft für die Balz beeinflussen und dadurch auch einen fördernden Einfluß auf den Auslösemechanismus für die Balz besitzen, aber keine Signalreize, die zur Auslösung von Balzverhalten führen. 
Heft 3

\section{Zusammenfassung}

Im Gegensatz zu optischen Komponenten des Auslösers „Weibchen“ ist die Balz männlicher Zebrafinken durch reinen akustischen Kontakt mit dem $\$$ nicht auslösbar. Akustischer Kontakt erhöht aber den Gesamterregungspegel des ${ }^{*}$ und führt im Vergleich zur Darbietung rein optischer Attrappen zu einer Intensivierung der Balz.

\section{Summary}

Influence of acoustic stimuli on courtship behaviour of male Zebra Finches

Courtship behaviour of male zebra finches is elicited by the presence of stuffed dummies, but not by acoustic contact with a female. Acoustic contart, however, enhances the arousal of the male and leads to a higher frequency of courtship behaviour if compared with experiments, where solely optic components of the releaser "female" are offered.

\section{Literatur}

Bischof, H. J. (1980): Reaktionen von Zebrafinken- $q$ auf zweidimensionale Attrappen: Einfluß von Reizqualität und Prägung. J. Orn. 121: 288-290. Ders. (1983): Der Balzgesang des Zebrafinken: Steuerung durch reizabhängige und reizunabhängige Faktoren. Verh. Dtsch. Zool. Ges. 1983: 186. - Ders., J. BöHNER \& R. SossinKa (1981): Influence of External Stimuli on the Quality of Song of the Zebra Finch (Taeniopygia guttata castanotis GoulD). Z. Tierpsychol. 57: 261-267. - BuchHoltz, Ch. (1982): Grundlagen der Verhaltensphysiologie. Vieweg, Braunschweig 1982. CARYL, P. G. (1981): The relationship between the motivation of directed and undirected song in the Zebra Finch. Z. Tierpsychol.57: 37-50. - Garson, P.J., J. DunN, C. J. WaLTON \& P. A. SHAW (1980): Stimuli eliciting courtship from domesticated Zebra Finches. Anim. Behav. 28: 1184-1187. - IMMELMANN, K. (1959): Experimentelle Untersuchungen über die biologische Bedeutung artspezifischer Merkmale beim Zebrafinken (Taeniopygia guttata castanotis Gould) . Zoo. Jb. 86: 438 - 593. - Ders. (1962): Beiträge zu einer vergleichenden Biologie australischer Prachtfinken (Spermestidae). Zool. Jb. Syst. Bd. 90: 1-196. MorRIs, D. (1954): The reproductive behaviour of the Zebra Finch. Behaviour 6; 271-322. - SiEGEL, S. (1976): Nichtparametrische statistische Methoden. Fachbuchhandlung für Psychologie, Verlagsabteilung, Frankfurt am Main. - SossrnKa, R. \& J. BöHner (1980): Song types in the Zebra Finch Poephila guttata castanotis. Z. Tierpsychol. 53; 123-132.

Anschrift des Verfassers: Fakultät Biologie, Lehrstuhl für Verhaltensphysiologie, Universität Bielefeld, Postfach 86 40, D-4800 Bielefeld 1. 